\title{
High-speed photothermal off-resonance atomic force microscopy reveals assembly routes of centriolar scaffold protein SAS-6
}

\author{
Adrian P. Nievergelt $\circledast^{1,3}$, Niccolò Banterle ${ }^{2,3}$, Santiago H. Andany', Pierre Gönczy ${ }^{2}$ and Georg E. Fantner®1*
}

\begin{abstract}
The self-assembly of protein complexes is at the core of many fundamental biological processes ${ }^{1}$, ranging from the polymerization of cytoskeletal elements, such as microtubules ${ }^{2}$, to viral capsid formation and organelle assembly ${ }^{3}$. To reach a comprehensive understanding of the underlying mechanisms of self-assembly, high spatial and temporal resolutions must be attained. This is complicated by the need to not interfere with the reaction during the measurement. As self-assemblies are often governed by weak interactions, they are especially difficult to monitor with high-speed atomic force microscopy (HS-AFM) due to the non-negligible tip-sample interaction forces involved in current methods. We have developed a HS-AFM technique, photothermal off-resonance tapping (PORT), which is gentle enough to monitor self-assembly reactions driven by weak interactions. We apply PORT to dissect the self-assembly reaction of SAS-6 proteins, which form a nine-fold radially symmetric ring-containing structure that seeds the formation of the centriole organelle. Our analysis reveals the kinetics of SAS- 6 ring formation and demonstrates that distinct biogenesis routes can be followed to assemble a nine-fold symmetrical structure.
\end{abstract}

Many biological structures are assembled and disassembled in a dynamic fashion to fulfil their function. Such dynamicity is often facilitated by low binding energies, as evidenced by relatively high dissociation constants $\left(K_{\mathrm{d}}\right)$, for instance of $0.8 \mu \mathrm{M}$ for the $\alpha \beta$-tubulin complex ${ }^{4}$ or of $10 \mu \mathrm{M}$ for the human immunodeficiency virus capsid homodimers ${ }^{5}$. The resulting geometry of protein assemblies, for example the length of microtubules, often depends on the corresponding assembly/disassembly kinetics. To understand the biogenesis of macromolecular structures, it is essential to observe such kinetics in real time with single-molecule resolution. HS-AFM in the amplitude-modulation mode, also known as the HS-tapping mode, is a powerful tool to study the dynamics of single proteins ${ }^{6}$ or of tightly bound protein assemblies ${ }^{7}$. Striking examples include pioneering studies on the actin-myosin complex ${ }^{6}$, which has a $K_{\mathrm{d}}$ of $0.5-10 \mathrm{nM}$ (ref. ${ }^{8}$ ), or of bacteriorhodopsin trimers embedded in a membrane ${ }^{9}$. However, the use of high-speed amplitude-modulation atomic force microscopy (HS-AM-AFM) to analyse the assembly dynamics of weaker interactions, with dissociation constants in the micromolar regime or above, has proved difficult.

A striking example of a weak interaction at the root of an important self-assembling structure occurs between homodimers of SAS-6, a scaffold protein required for centriole assembly in organisms from algae to humans ${ }^{10,11}$. Centrioles are nine-fold radially symmetric microtubule-based organelles that play critical roles in eukaryotic cell physiology ${ }^{12}$. Homodimers of SAS- 6 proteins undergo a higherorder oligomerization through a weak interaction mediated by their globular head domain, with a dissociation constant of $\sim 60 \mu \mathrm{M}$ (refs ${ }^{10,11}$ ). These higher-order oligomers form nine-fold symmetric ring-like structures that initiate centriole formation in vivo and impart the signature nine-fold radial symmetry to the entire organelle ${ }^{13,14}$ (Fig. 1a). Recombinantly produced homodimers of the Chlamydomonas reinhardtii SAS- 6 protein truncated of its C-terminal domain (hereafter referred to as CrSAS-6 (Methods)) can form structures that contain nine-fold radially symmetrical rings also in vitro ${ }^{10}$, which can be observed after their assembly using transmission electron microscopy ${ }^{10}$ or $\mathrm{AFM}^{15,16}$ (Fig. $1 \mathrm{~b}$ and Supplementary Fig. 5). However, the dynamics of the SAS-6 ring assembly have not been resolved to date, which precludes a thorough understanding of the mechanisms at the root of centriole biogenesis.

We attempted to image CrSAS-6 ring assembly dynamics with HS-AM-AFM (Fig. 1c,d) on our home-built instrument described in Methods, as well as on a commercial HS-AFM (data not shown). However, we found that whereas some homodimers associated to form intermediate assembly states (Fig. 1c), no full rings were formed in the imaging area (Fig. 1d, dashed box and green inset, Supplementary Video 1, Supplementary Fig. 2 and Supplementary Table 1). By contrast, we found that full-ring structures had formed in the regions that surround the area that had been imaged throughout the experiment (Fig. 1d, red inset, magenta circles). Similarly, imaging fully assembled rings with HS-AM-AFM leads to their disruption (Supplementary Figs. 1 and 3, and Supplementary Video 2). Together, these observations suggest that the tip-sample interaction during HS-AM-AFM imaging interferes with the assembly process and ring stability. AFM based on the force-distance curve ${ }^{17}$ (FD-AFM (PeakForce Tapping)) has been used previously to observe fully assembled SAS- 6 rings using $45-65 \mathrm{pN}$ force set points ${ }^{15,16}$. For imaging biological samples in solution, tip forces have been reported to be significantly less than those in AM-AFM when using FD-AFM with a cycle-to-cycle feedback ${ }^{18-21}$. We refer to this general AFM class as off-resonance tapping (ORT), to make a clear distinction with resonant modes, such as AM-AFM or frequency modulation AFM, and to avoid confusion with existing commercial implementations of force-distance-based imaging modes. However, the currently available ORT methods are inherently too slow to measure the dynamics of self-assembly reactions like that of SAS- 6 proteins. Here, we present an advanced ORT imaging mode using photothermal actuation ${ }^{22}$, referred to as photothermal off-resonance tapping

'Laboratory for Bio- and Nano-Instrumentation, Swiss Federal Institute of Technology Lausanne (EPFL), Lausanne, Switzerland. ${ }^{2}$ Swiss Institute for Experimental Cancer Research (ISREC), School of Life Sciences, Swiss Federal Institute of Technology Lausanne (EPFL), Lausanne, Switzerland.

${ }^{3}$ These authors contributed equally to this work: Adrian P. Nievergelt, Niccolò Banterle. *e-mail: georg.fantner@epfl.ch 
a

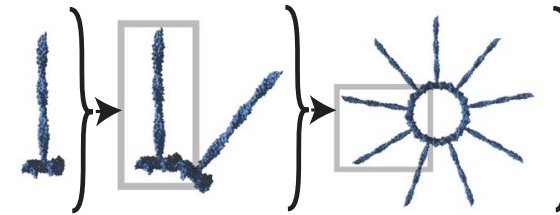

$1 \times$

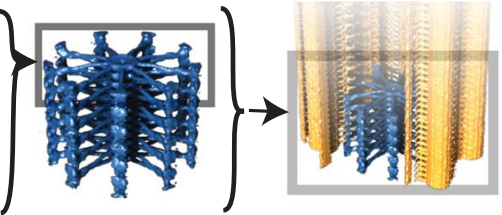

Cartwheel

Centriole
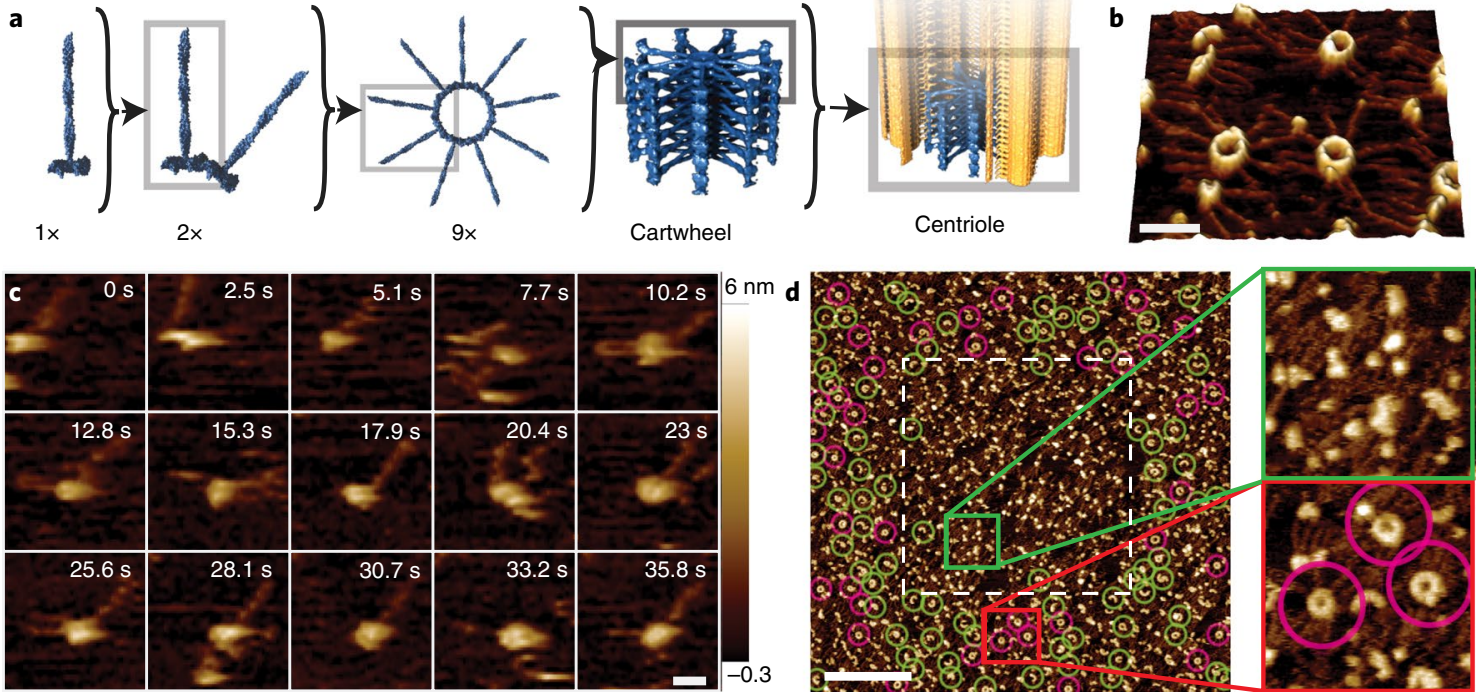

Fig. 1 | Assembly of the centriolar scaffolding protein CrSAS-6 cannot be observed with conventional HS-AM-AFM. a, A single CrSAS-6 homodimer $(1 \times)$ is composed of a pair of coiled-coil domains and a pair of globular head domains, as well as of unstructured C-terminal regions (not represented). CrSAS-6 homodimers interact through their head domains and form intermediates ( $2 \times$ ), which eventually assemble into nine-fold symmetric rings ( $9 \times$ ) that subsequently stack to form the centriolar cartwheel. The cartwheel acts as a scaffold for the formation of a new centriole through the recruitment of more peripheral components, which include microtubules (yellow). b. High-resolution AFM of equilibrated CrSAS-6 in vitro assemblies reveal the circular protein ring and the attached spokes (coiled-coil domains). Scale bar, $50 \mathrm{~nm}$. c, HS-AM-AFM frame grabs show SAS-6 homodimers forming intermediate assemblies, which fall apart when scanned multiple times. No formation of the full rings can be observed. Scale bar, 10 nm; $z$ scale, $-0.3-6$ nm. d, The region imaged with HS-AM-AFM (dashed white square) during assembly is clearly distinct from the surrounding area. Assemblies that form more than half rings (green circles) and full rings (magenta circles) are marked and present essentially only outside the imaging area (dashed box, where essentially only protein clumps are observed). Scale bar, $300 \mathrm{~nm}$; $z$ scale as in c.

(PORT), which enables force-distance curves up to two orders of magnitude faster than in conventional methods.

In ORT, the achievable imaging speed is limited by the rate at which the cantilever tip can be moved up and down relative to the sample in a controlled manner. In conventional ORT implementations, this motion is performed by a piezo that moves the cantilever chip or the sample (Fig. 2a). Moving such a relatively large mass limits the forcecurve rate to a few kilohertz for most scanners and up to $32 \mathrm{kHz}$ for advanced designs ${ }^{23}$. In contrast, when utilizing photothermal actuation $^{22}$ with the bimorph effect in a coated cantilever, the mass that needs to be moved comprises only the cantilever and the tip (Fig. 2b). This allows ramp speeds of several hundred kilohertz (Fig. 2d), limited only by the resonance of the cantilever (Fig. 2c). As the cantilever is being actuated directly, the measured cantilever deflection consists of the tip-sample force interaction of interest, and a background motion (Fig. 2b, left inset). The tip-sample interaction can be determined by recording the background motion slightly above the surface and subtracting it in real time from the ORT curves when imaging on the actual surface (Fig. 2b (centre inset), Fig. 3a and Supplementary Section 3). This background corrected time trace can then be reconstructed into a tip-sample force curve (Fig. 2b, right inset).

To determine the minimally achievable tip-sample forces in PORT using a commercial $8 \times 2 \mu \mathrm{m}$ high-speed cantilever $\left(k=0.22 \mathrm{~N} \mathrm{~m}^{-1}, f_{\text {res }}^{\text {fluid }}=505 \mathrm{kHz}, Q=1.5\right.$ (BioLever Fast BLAC10DS (Olympus))), we recorded highly averaged time traces of the tip-sample interaction at a $100 \mathrm{kHz}$ ORT rate, and a $20 \mathrm{~nm}$ amplitude in imaging buffer on freshly cleaved mica surfaces (Methods). Neglecting the viscoelastic behaviour of the sample, the force exerted by the cantilever on the sample consists of two components: the static force given by the cantilever deflection and the dynamic impact force due to the abrupt deceleration of the moving tip ${ }^{19}$. The dynamic impact force is given by Newton's law as:

$$
F_{\text {dyn }}=m_{\text {eff }} a
$$

with $m_{\text {eff }}=k /\left(2 \pi f_{\text {res }}\right)^{2}$ being the effective mass ${ }^{19}$ and $a$ the acceleration of the cantilever. In conventional ORT, the dynamic force can usually be neglected because of the relatively low velocity of the tip at impact. As the rate, and consequently the impact velocity, increases with PORT, the impact force of $\sim 150 \mathrm{pN}$ (Fig. 3b) can easily exceed the static force set point of $80 \mathrm{pN}$ (Fig. 3a). In the highly damped case of imaging in liquid with small, soft cantilevers, the cantilever approaches the critical damping. Then, assuming Hertzian mechanics, the maximum impact force $P^{\star}$ scales with:

$$
P^{\star} \propto\left(\frac{f_{\mathrm{m}}}{f_{\text {res }}}\right)^{6 / 5}
$$

where $f_{\mathrm{m}}$ is the measurement frequency and $f_{\text {res }}$ the cantilever resonance frequency ${ }^{24}$ (see Supplementary Section 5 for details and derivation). This equation does not make any assumption about the feedback mode, and is therefore also valid for AM-AFM measurements in highly damped environments.

To compare the tip-sample forces in high-speed photothermal off-resonance tapping (HS-PORT) with those in HS-AM-AFM, we recorded highly averaged time traces of the same cantilever deflection when exciting at resonance and feeding back on the amplitude. From this, we measured dynamic tip-sample forces of $\sim 800 \mathrm{pN}$ for HS-AM-AFM (Fig. 3c,d), compared to $\sim 150 \mathrm{pN}$ for HS-PORT (Fig. 3b). We calculated the kinetic energies of the cantilever at impact for HS-AM-AFM as $2.5 \times 10^{-19} \mathrm{~J}$ (Fig. 3c), and for HS-PORT as $1.12 \times 10^{-20} \mathrm{~J}$ for a $50 \mathrm{pN}$ set point (Fig. 3a). Moreover, we found that the tip-sample forces in HS-AM-AFM increase rapidly with increasingly aggressive set points (Fig. 3f,h), as previously established ${ }^{19,21,25}$. Very high set points $(>90 \%)$ can lead to parachuting artefacts in imaging, unless specialized instrumentation is used, such as gain-scheduling control ${ }^{26}$. By contrast, the impact force in HS-PORT scales more gradually with the set point (Fig. 3e,i). 
a

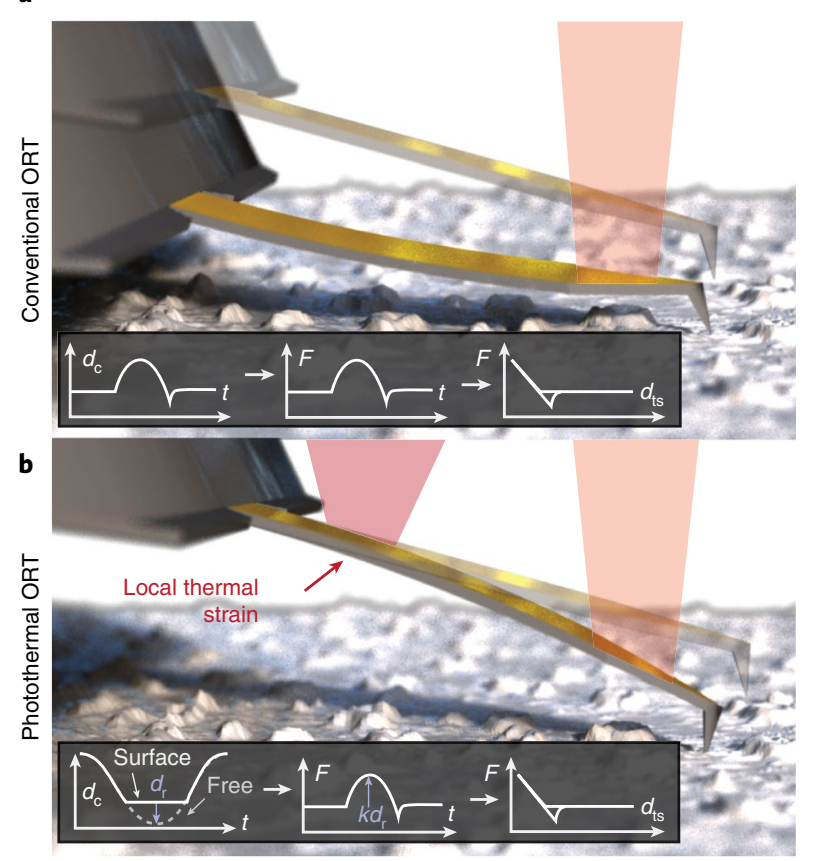

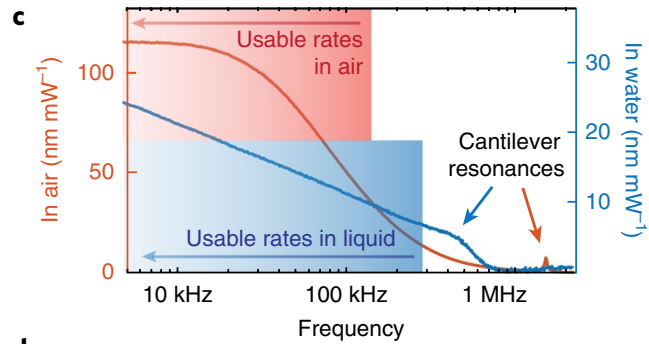

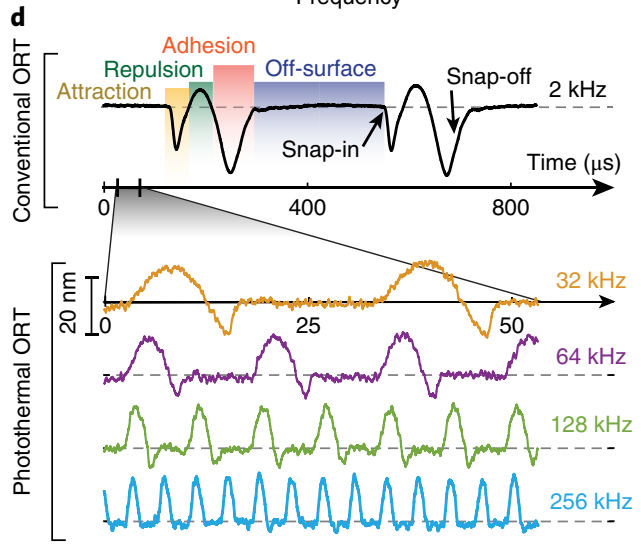

Fig. 2 | Basic principle of HS-PORT. a, Conventional ORT moves the cantilever or the sample to probe the surface with the cantilever tip. The large mass moved in this way by the piezo limits the measurement rate, and in turn the imaging speed. The observed cantilever deflection, $d_{c}$ (left inset) can be directly interpreted as a force (middle inset) and reconstructed into a force-distance curve (right inset). b, PORT uses laser-based heating to actuate the cantilever directly, which ensures that the mass in motion consists only of the cantilever and the tip. The resulting motion (left inset) is superimposed onto the surface interaction, but the force-time curve (middle inset) can be recovered by subtracting the reference motion, previously recorded away from the surface to get the restricted distance $d_{r}$. The force over time is then $k d_{r}$ where $k$ is the spring constant of the cantilever. A force-distance curve (force $F$ to tip-sample distance $d_{\mathrm{ts}}$ ) (right inset) can then be reconstructed in the same way as in conventional ORT. c, The photothermal drive response of an $8 \times 2 \mu \mathrm{m}$ high-speed cantilever has a large quasistatic bending region below $300 \mathrm{kHz}$, which is used for PORT actuation. $\mathbf{d}$, The signal observed in ORT has the characteristic heartbeat shape of an adhesive snap-in, a repulsive motion of the cantilever in contact, followed by an adhesive snap-off from the surface (top). In PORT (bottom), the same curves can be reconstructed after subtracting the bending induced by photothermal heating. The reduced mass in motion allows an increase in measurement frequency $f_{\mathrm{m}}$ to $256 \mathrm{kHz}$.

Equation (2) suggests that the dynamic force component scales with the ratio of $f_{\mathrm{m}}$ to the cantilever $f_{\text {res }}$. In the case of HS-AM-AFM, $f_{\mathrm{m}}$ and $f_{\text {res }}$ are inherently linked and practically equal, whereas in HS-PORT, $f_{\mathrm{m}}$ can be chosen freely, provided it is sufficiently below the first resonance of the cantilever ${ }^{27}$. In addition, resonance-based techniques require advanced electronics for single-cycle detection $^{28,29}$. We measured the dynamic forces for different ratios of $f_{\mathrm{m}}$ to $f_{\text {res }}$ (Fig. $3 \mathrm{~g}$ ). Fitting this curve with a constrained power law, we found an exponent of 1.3 (95\% confidence interval 1.22-1.40). That the measured exponent is slightly larger than the 1.2 predicted by the estimation of the Hertzian model suggests that, though the latter is a good first approximation, most likely other factors play a role as well. The future development of a full theoretical model for PORT, based on time-dependent, non-linear contact mechanics, could potentially describe the influence of other factors on the dynamic force, such as ORT amplitude and impact angle. However, the frequency-ratio dependence already provides an intuitive explanation as to why the measured tip-sample forces in HS-PORT are substantially lower than those in HS-AM-AFM.

In our discussion of AM-AFM, we limit ourselves to AM-AFM experiments in which the amplitude of the oscillation is higher than the decay lengths of the interaction forces ${ }^{30}$. By contrast, recent amplitude modulation $3 \mathrm{D}$ AFM measurements ${ }^{31}$ with very small oscillation amplitude do not suffer from this effect. However, they are less suited for observing dynamic processes due to the required independent $z$ motion overlaid on the tip oscillation.

With the advantages of PORT for gentle and rapid topological probing established, we proceeded to utilize this novel imaging mode to monitor the assembly of CrSAS-6 rings with a home-built AFM (Supplementary Sections 3 and 4) using a concentration of $\sim 61 \mathrm{nM}$, in the order of that estimated for the homologous protein HsSAS-6 in the cytoplasm of human cells at the onset of centriole assembly ${ }^{32,33}$. As shown in Fig. 4a, in contrast to the observations with HS-AM-AFM (Fig. 1c), we found that with HS-PORT there is no noticeable difference in the number of fully formed rings when the area imaged during assembly is compared with the surrounding regions. At sufficiently high force set points, the disruption of the formed rings can, nevertheless, be controllably induced (Supplementary Figs. 1-3). During the imaging, a manifold of distinct, highly dynamic self-assembly reactions was observed (as exemplified in Fig. 4b and Supplementary Videos 3-5). As a first step towards characterizing the dynamics of CrSAS-6 ring assembly, we set out to determine the time needed to assemble a full CrSAS-6 ring, and thus estimate how quickly the process could occur in vivo. As shown in Fig. 4c, HS-PORT imaging revealed that CrSAS- 6 rings assemble in 106 seconds on average. The canonical route that leads to ring assembly of CrSAS- 6 proteins is believed to entail the sequential addition of homodimers, from the initial single homodimer to the nine homodimers present in the ring structure. Strikingly, HS-PORT imaging uncovered that, whereas such an assembly route is, indeed, observed (Fig. 4d (top row) and Supplementary Video 6), other assembly routes exist as well. These include the merging of two higher-order multimeric units (Fig. $4 \mathrm{~d}$ (middle row) and Supplementary Video 7), and interlinked halfcircle structures that undergo topological rearrangement and relaxation into a ring (Fig. 4d (bottom row) and Supplementary Video 8). 

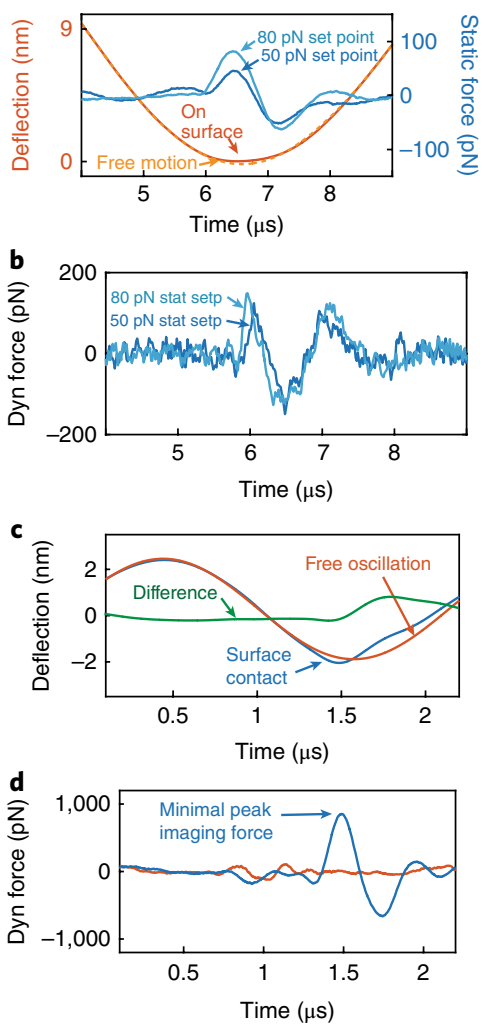

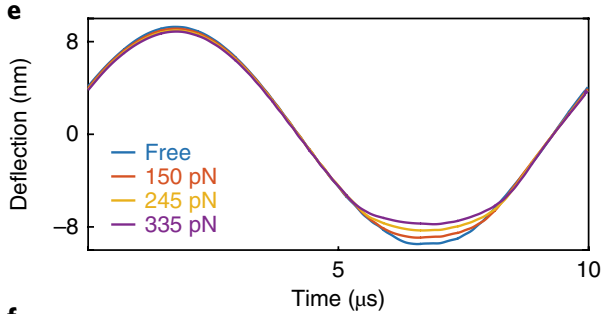

f

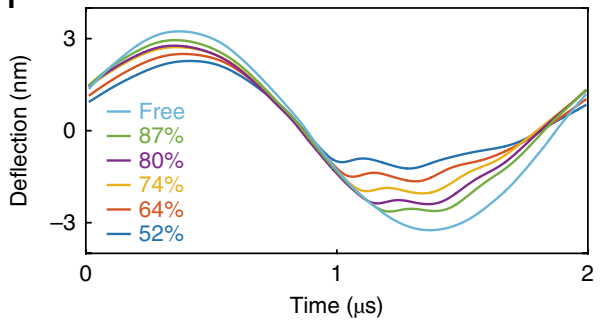

g

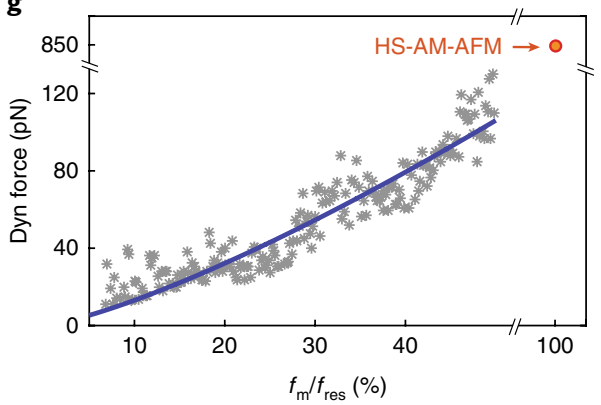

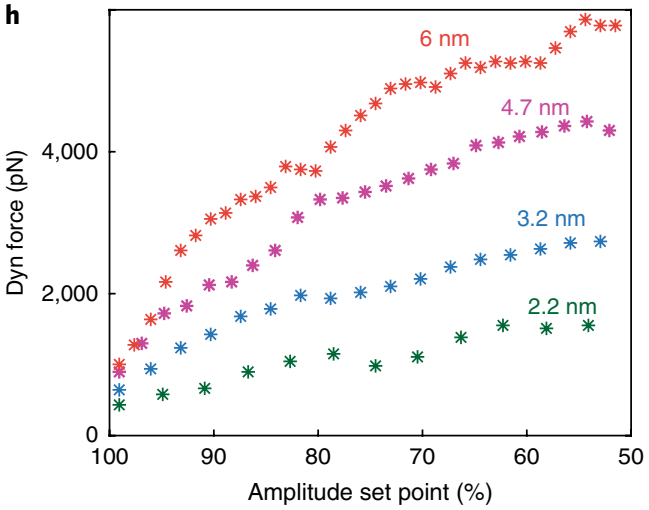

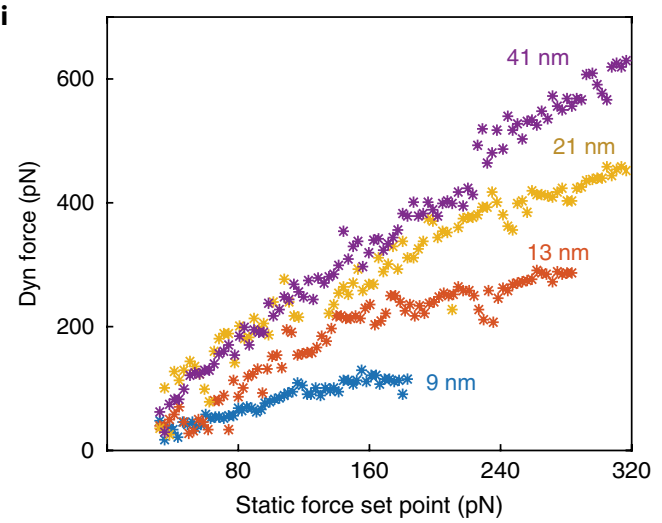

Fig. 3 | Imaging forces on mica in liquid comparing HS-AM-AFM and HS-PORT. a, In HS-PORT $\left(f_{\mathrm{m}}=100 \mathrm{kHz}\right)$, the cantilever actuation is visible in the detector (orange dashed line) and changes on interacting with the surface (red line). The difference between the two signals is proportional to the static force onto the sample, which is used for topographical feedback. Very small static set-point (stat setp) forces ( $50 \mathrm{pN}$ and $80 \mathrm{pN}$ ) can be used. b. The sudden impact of the cantilever onto the surface causes a short dynamic (Dyn) force pulse. This pulse, depending on the amplitude and actuation rate, can surpass the set-point force $(100 \mathrm{pN}$ for the $50 \mathrm{pN}$ static set point and $160 \mathrm{pN}$ for the $80 \mathrm{pN}$ static set point). c, In HS-AM-AFM (f res $=505 \mathrm{kHz}$, $f_{m}=480 \mathrm{kHz}$ ), using a set-point reduction of only $0.2 \mathrm{~nm}$ from a free amplitude of $2 \mathrm{~nm}$ causes a very fast non-linear impact with the surface (compare the deflection trace on the surface (blue) with the free motion trace (red); the difference is in green) followed by an inertia-limited recovery to the steadystate amplitude. d, The dynamic impact in HS-AM-AFM causes a short force pulse of $850 \mathrm{pN}$ even for a very gentle amplitude set point. e, In PORT, the deflection trace is clipped at the low end, which varies with the used set point. $\mathbf{f}$, In HS-AM-AFM, the impact of the cantilever on the surface causes a large clipping of the sinusoidal motion, but only a small change in the amplitude shortly before impact. $\mathbf{g}$, The dynamic force in PORT scales approximately with the ratio of the $f_{\mathrm{m}}$ to the cantilever $f_{\text {res }}$ as $\left(f_{\mathrm{m}} / f_{\text {res }}\right)^{1.3}$ (blue fit). In HS-AM-AFM, this ratio is fixed and the dynamic forces are significantly higher (red circle to the right of the axis break). $\mathbf{h}$, In HS-AM-AFM, the impact force increases rapidly even at set points close to the free amplitude. $\mathbf{i}$, In HS-PORT $\left(f_{m}=100 \mathrm{kHz}\right)$, the dynamic impact force scales gradually with the static force set point and increases with larger amplitudes. All the cantilever excitation was performed via a photothermal drive.

The existence of different assembly routes demonstrates that the SAS-6 assembly process is not deterministic, but instead somewhat plastic. Such plasticity lends itself better to regulation, which includes regulation by partner proteins in the cellular context, and offers a greater potential for an efficient repair.

The use of photothermal actuation for off-resonance tapping enabled us to probe the assembly reactions governed by weak protein interactions, as exemplified in the case of SAS- 6 proteins. Traditionally, photothermal actuation is used exclusively to excite cantilevers at resonance, in part because the photothermal drive efficiency is limited for normal sized cantilevers, which results in a small achievable offresonance actuation range. For small cantilevers, such as the AC-10, however, the drive efficiency is much higher because the thickness ratio of the coating to cantilever base material is more beneficial ${ }^{34}$, and thereby allows sufficient ORT amplitudes (Fig. 2d). The use of photothermal excitation for ORT allowed us to increase the speed by approximately two orders of magnitude using commercially available cantilevers. As the dynamic tip-sample force decreases with increased cantilever $f_{\text {res }}$, to develop even smaller cantilevers will directly increase the imaging speed as it decreases the impact forces (Fig. 3g).
Factors other than the inherent tip-sample-interaction force could also play a role in HS-PORT measurements being better suited than HS-AM-AFM for imaging CrSAS-6 assemblies. To obtain acceptably small forces in AM-AFM, small amplitudes should be used. However, even at amplitudes of $1-4 \mathrm{~nm}$ and set-point amplitudes of $90 \%$, we were not able to image without impairing self-assembly (Supplementary Figs. 2 and 4). Due to the finite speed of the feedback loop, the sample is subjected to lateral forces on a change in topography, even when imaging molecular structures of a few nanometres in height. These forces act in plane with the weak self-assembly forces, and therefore directly affect the dissociation probability. In PORT, a larger ramp amplitude can be chosen with acceptably small forces still maintained, because of the lower $f_{\mathrm{m}} / f_{\text {res }}$ ratio.

In our current implementation of HS-PORT, heating is minimized by using a pulsed operation of the drive laser rather than a continuous sinusoidal actuation (Supplementary Fig. 10), which more than halves the average heat on the cantilever. The resulting cantilever motion is sufficiently sinusoidal on the down part of the curve such that the assessment of the impact forces is still applicable. 


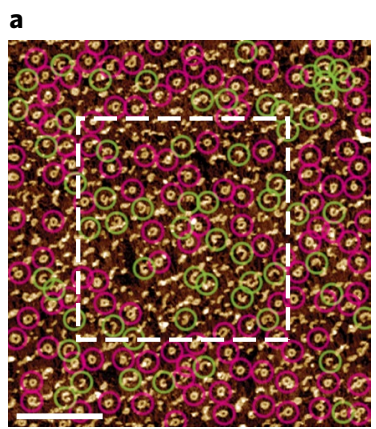

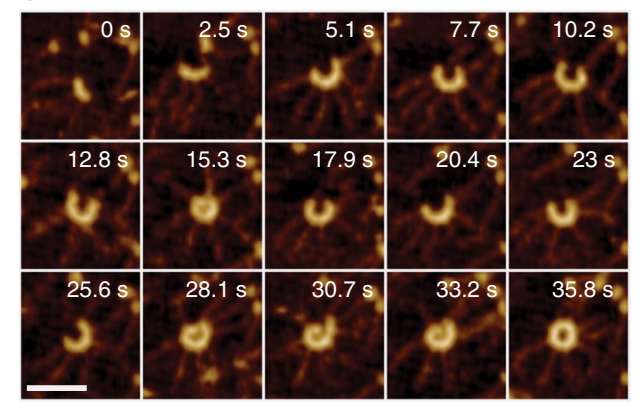

c

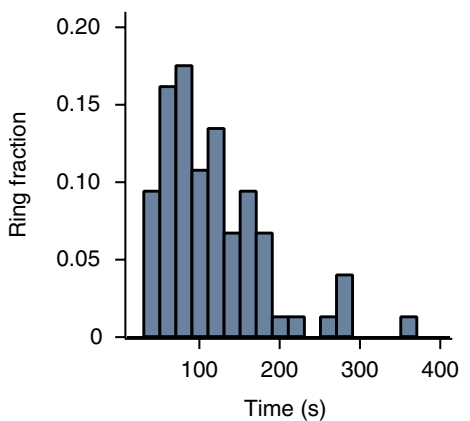

d
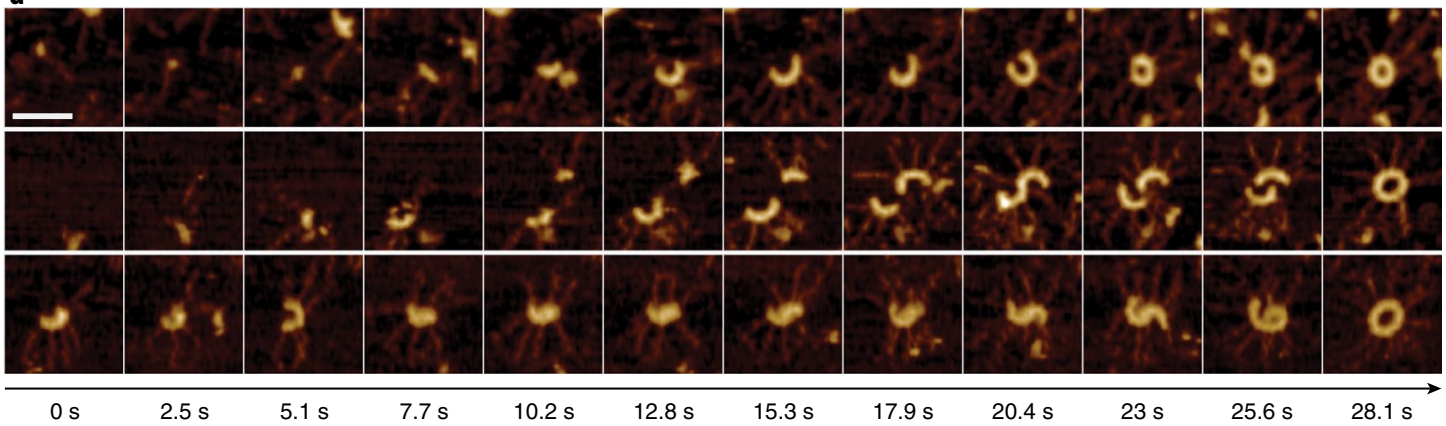

Fig. 4 | Imaging and analysis of CrSAS-6 self-assembly using HS-PORT. a, Comparison of the area imaged during assembly (dashed white line) with the surrounding region shows no significant difference in the density of higher-order assemblies (green circles) or full rings (magenta circles). Scale bar, $300 \mathrm{~nm} ; \mathrm{z}$ scale, $6.3 \mathrm{~nm}$ (as in Fig. 1). b, HS-PORT allows the recording of a full assembly process from the initial stages through a diversity of intermediate assembly stages, and ends in a closed ring. Scale bar, $50 \mathrm{~nm} ; z$ scale, $-0.3-6 \mathrm{~nm}$ (as in Fig. 1). c, A histogram of the time from the initial nucleation to the fully formed CrSAS- 6 rings shows an average time of formation of $106 \mathrm{~s}$. (s.d., $66 \mathrm{~s}$ ) measured over 75 individual assemblies. d, HS-PORT reveals distinct assembly routes. Though rings can be formed by the sequential addition of homodimers (top), the merging of two large fragments can also lead to the formation of a ring (middle). Moreover, in some instances, intermediate metastable states can undergo a conformational change that leads to ring formation (bottom). Scale bar, $50 \mathrm{~nm}$; $z$ range, $-0.3-6 \mathrm{~nm}$ (as in Fig. 1).

In the future, optimizing the cantilever coating materials and thicknesses will further improve the drive efficiency, which will allow a larger actuation range with reduced heating.

Using HS-PORT allowed us to monitor the molecular dynamics of SAS- 6 self-assembly. As the mica-CrSAS- 6 interaction most probably influences the reaction kinetics, the measured assembly time should be considered as an order of magnitude indication rather than an exact representation of the in vivo assembly time. Nevertheless, to understand the assembly kinetics of CrSAS-6 rings in vitro could help shed light on the conditions relevant to cartwheel assembly in vivo, which occurs exclusively on surfaces $^{35}$, as is the case in the present cell-free assay. Moreover, multiple rings of SAS- 6 proteins stack to create the cartwheel structure in vivo, probably assisted by other molecular partners ${ }^{35}$. How these molecular partners contribute to cartwheel formation is not yet understood. In the future, HS-PORT measurements performed in the presence of such molecular partners will further help understand this crucial self-assembly mechanism that occurs in eukaryotic cells.

\section{Methods}

Methods, including statements of data availability and any associated accession codes and references, are available at https://doi. org/10.1038/s41565-018-0149-4.

Received: 20 June 2017; Accepted: 19 April 2018; Published online: 21 May 2018

\section{References}

1. Kushner, D. J. Self-assembly of biological structures. Bacteriol. Rev. 33, 302-345 (1969).
2. Mandelkow, E. M. Microtubule dynamics and microtubule caps: a time-resolved cryo-electron microscopy study. J. Cell Biol. 114, 977-991 (1991).

3. Cameron, J. C., Wilson, S. C., Bernstein, S. L. \& Kerfeld, C. A. Biogenesis of a bacterial organelle: the carboxysome assembly pathway. Cell 155, 1131-1140 (2013).

4. Detrich, H. W.., \& Williams, R. C.. Reversible dissociation of the $\alpha \beta$ beta dimer of tubulin from bovine brain. Biochemistry 17, 3900-3907 (1978).

5. Garzón, M. T. et al. The dimerization domain of the HIV-1 capsid protein binds a capsid protein-derived peptide: a biophysical characterization. Protein Sci. 13, 1512-1523 (2004).

6. Kodera, N., Yamamoto, D., Ishikawa, R. \& Ando, T. Video imaging of walking myosin $\mathrm{V}$ by high-speed atomic force microscopy. Nature 468 , 72-76 (2010).

7. Preiner, J. et al. IgGs are made for walking on bacterial and viral surfaces. Nat. Commun. 5, 4394 (2014).

8. Oshima, H., Hayashi, T. \& Kinoshita, M. Statistical thermodynamics for actin-myosin binding: the crucial importance of hydration effects. Biophys. J. 110, 2496-2506 (2016).

9. Shibata, M., Yamashita, H., Uchihashi, T., Kandori, H. \& Ando, T. High-speed atomic force microscopy shows dynamic molecular processes in photoactivated bacteriorhodopsin. Nat. Nanotech. 5, 208-212 (2010).

10. Kitagawa, D. et al. Structural basis of the 9 -fold symmetry of centrioles. Cell 144, 364-375 (2011).

11. van Breugel, M. et al. Structures of SAS-6 suggest its organization in centrioles. Science 331, 1196-1199 (2011).

12. Bornens, M. The centrosome in cells and organisms. Science 335, 422-426 (2012).

13. Hirono, M. Cartwheel assembly. Philos. Trans. R. Soc. B 369, 20130458 (2014).

14. Strnad, P. \& Gönczy, P. Mechanisms of procentriole formation. Trends Cell Biol. 18, 389-396 (2008).

15. Pfreundschuh, M., Alsteens, D., Hilbert, M., Steinmetz, M. O. \& Müller, D. J. Localizing chemical groups while imaging single native proteins by high-resolution atomic force microscopy. Nano Lett. 14, 2957-2964 (2014).

16. Hilbert, M. et al. SAS-6 engineering reveals interdependence between cartwheel and microtubules in determining centriole architecture. Nat. Cell Biol. 18, 393-403 (2016). 
17. Rosa-Zeiser, A., Weilandt, E., Hild, S. \& Marti, O. The simultaneous measurement of elastic, electrostatic and adhesive properties by scanning force microscopy: pulsed-force mode operation. Meas. Sci. Technol. 8 , 1333-1338 (1997).

18. Ortega-Esteban, A. et al. Minimizing tip-sample forces in jumping mode atomic force microscopy in liquid. Ultramicroscopy 114, 56-61 (2012).

19. Xu, X., Carrasco, C., de Pablo, P. J., Gomez-Herrero, J. \& Raman, A. Unmasking imaging forces on soft biological samples in liquids when using dynamic atomic force microscopy: a case study on viral capsids. Biophys. J. 95, 2520-8 (2008)

20. Ashby, P. D. Gentle imaging of soft materials in solution with amplitude modulation atomic force microscopy: Q control and thermal noise. Appl. Phys. Lett. 91, 254102 (2017).

21. Kumar, B., Pifer, P. M., Giovengo, A. \& Legleiter, J. The effect of set point ratio and surface Young's modulus on maximum tapping forces in fluid tapping mode atomic force microscopy. J. Appl. Phys. 107, 044508 (2010).

22. Ratcliff, G. C., Erie, D. A. \& Superfine, R. Photothermal modulation for oscillating mode atomic force microscopy in solution. Appl. Phys. Lett. 72, 1911-1913 (1998).

23. Nievergelt, A. P., Erickson, B. W., Hosseini, N., Adams, J. D. \& Fantner, G. E. Studying biological membranes with extended range high-speed atomic force microscopy. Sci. Rep. 5, 11987 (2015).

24. Johnson, K. L. Contact Mechanics (Cambridge Univ. Press, Cambridge, 1985)

25. Guzman, H. V., Perrino, A. P. \& Garcia, R. Peak forces in high-resolution imaging of soft matter in liquid. ACS Nano 7, 3198-3204 (2013).

26. Kodera, N., Sakashita, M. \& Ando, T. Dynamic proportional-integraldifferential controller for high-speed atomic force microscopy. Rev. Sci. Instrum. 77, 83704 (2006).

27. Amo, C. A. \& Garcia, R. Fundamental high-speed limits in singlemolecule, single-cell, and nanoscale force spectroscopies. ACS Nano 10, 7117-7124 (2016).

28. Ando, T. et al. A high-speed atomic force microscope for studying biological macromolecules. Proc. Natl Acad. Sci. USA 98, 12468-12472 (2001).

29. Schlecker, B. et al. Single-cycle-PLL detection for real-time FM-AFM applications. IEEE Trans. Biomed. Circuits Syst. 8, 206-215 (2014).

30. Garcia, R. \& Herruzo, E. T. The emergence of multifrequency force microscopy. Nat. Nanotech. 7, 217-226 (2012).

31. Martin-Jimenez, D., Chacon, E., Tarazona, P. \& Garcia, R. Atomically resolved three-dimensional structures of electrolyte aqueous solutions near a solid surface. Nat. Commun. 7, 12164 (2016).

32. Keller, D. et al. Mechanisms of HsSAS-6 assembly promoting centriole formation in human cells. J. Cell Biol. 204, 697-712 (2014).
33. Bauer, M., Cubizolles, F., Schmidt, A. \& Nigg, E. A. Quantitative analysis of human centrosome architecture by targeted proteomics and fluorescence imaging. EMBO J. 35, 2152-2166 (2016).

34. Zhang, Y., Zhang, Y. \& Marcus, R. B. Thermally actuated microprobes for a new wafer probe card. J. Micro. Syst. 8, 43-49 (1999).

35. Gönczy, P. Towards a molecular architecture of centriole assembly. Nat. Rev. Mol. Cell Biol. 13, 425-435 (2012).

\section{Acknowledgements}

The authors thank C. Brillard, J. D. Adams and G. Hatzopoulos for assistance. We also thank F. Johann and J. Lopez from Oxford Instruments for help during the measurements with their Cypher VRS microscope. We thank the EPFL workshops ATPR and ATMX for the fabrication of research equipment. This work was funded by the European Union's Seventh Framework Programme FP7/2007-2013 under grant agreement 286146, the European Union's Seventh Framework Programme FP7/2007-2013/ERC grant agreements 307338 (to G.E.F.) and 340227 (to P.G.), and the European Union H2020 Framework Programme for Research \& Innovation (2014-2020), ERC-2017-CoG, InCell, Project number 773091 (to G.E.F). N.B. was supported initially by a grant from the ERC to P.G. (AdG 340227), and then by the EPFL Fellows postdoctoral fellowship program funded by the European Union's Horizon 2020 Framework Programme for Research and Innovation (Grant agreement 665667, MSCA-COFUND).

\section{Author contributions}

A.P.N. and N.B. contributed equally to this work. A.P.N. designed and built the instrument, performed experiments, analysed data and wrote the paper. N.B. prepared samples, performed experiments, analysed data and wrote the paper. S.H.A. built the instrumentation. P.G. conceived the experiments and wrote the paper. G.E.F. designed the instruments, conceived experiments and wrote the paper. All the authors discussed the results and commented on the manuscript.

\section{Competing interests}

The authors declare no competing financial interests.

\section{Additional information}

Supplementary information is available for this paper at https://doi.org/10.1038/ s41565-018-0149-4

Reprints and permissions information is available at www.nature.com/reprints. Correspondence and requests for materials should be addressed to G.E.F. Publisher's note: Springer Nature remains neutral with regard to jurisdictional claims in published maps and institutional affiliations. 


\section{Methods}

Protein expression and purification. The Bld12_NL truncated version of the CrSAS-6/Bld 12 protein $^{10}(58 \mathrm{kDa})$ was expressed in the Escherichia coli strain BL21(DE3) (Stratagene). Bacteria were grown at $37^{\circ} \mathrm{C}$ to an $A_{600}$ of 0.7 in Luria broth supplied with kanamycin. After induction with $0.5 \mathrm{mM}$ IPTG (isopropyl $\beta$-D-1-thiogalactopyranoside), protein expression was performed overnight at $20^{\circ} \mathrm{C}$. Bacterial cells were collected by centrifugation at $4,000 \mathrm{~g}$ for $10 \mathrm{~min}$ (JLA-9.1000 (Beckman Coulter)). Lysis was achieved by sonication of the resuspended bacterial pellet in $50 \mathrm{mM}$ Tris $\mathrm{pH} 7.5$ and $500 \mathrm{mM} \mathrm{NaCl}$ supplemented with proteases inhibitors (Complete EDTA-free (Roche)). The cellular debris was removed by spinning for $1 \mathrm{~h}$ at $12,000 \mathrm{~g}$ at $4{ }^{\circ} \mathrm{C}$ (JA 25.50 (Beckman Coulter)). The cleared lysate was then subjected to nickel purification with an HisTrap HP Ni ${ }^{2+}-$ Sepharose column (GE Healthcare) at $4^{\circ} \mathrm{C}$ and further purified by gel filtration with a HiLoad Superdex 200 16/60 column (GE Healthcare) equilibrated in $20 \mathrm{mM}$ Tris-HCl pH 7.4 supplemented with $150 \mathrm{mM} \mathrm{KCl}$. The sample was concentrated with an Amicon ultrafilter with a $10 \mathrm{kDa}$ cut-off, aliquoted and stored at $-80^{\circ} \mathrm{C}$.

PORT implementation. Measurement hardware. The HS-AFM consists of a custom-built head, scanner and controller (Supplementary Figs. 6 and 7) combined with a commercial piezo amplifier (Techproject), a MultiMode 8 AFM base and a NanoScope 5 controller (both Bruker) for the sample approach. A spring-based vibration isolator (BM4 (Minus-K)) provided isolation from the ambient vibration. The home-built head is compatible with small cantilevers (spot size $<1.5 \mu \mathrm{m} \times 3 \mu \mathrm{m}$ ) and has a possibility for an in-built photothermal drive. The head used in this work is an upgraded version from what we presented previously ${ }^{36}$, and features a smaller spot size and improved mechanics. The scanner is a flexure-based high-speed scanner with a $1.8 \mu \mathrm{m} \times 1.8 \mu \mathrm{m} \times 2 \mu \mathrm{m}$ scan range with $\sim 100 \mathrm{kHz} z$ bandwidth that is compatible with the approach mechanism of the MultiMode base (Supplementary Section 3).

Photothermal off-resonance controller. A custom-made high-speed ORT controller that consisted of a scan engine and ORT feedback using photothermal drive was implemented in LabView and LabView FPGA on a PXI system (PXIe-1082 (National Instruments)) connected to the control computer via an MXI-e interface (PXIe-8370) (Supplementary Figs. 8-11). The scan engine runs on an R-series Virtex-5 (PXI-7851R), whereas the feedback is implemented on a Kintex-7 (PXIe7975R) with a d.c.-coupled RF I/O-module (NI 5782 (National Instruments)). Supplementary Section 4 gives a detailed description of the controller implementation.

Preparation of SAS-6 samples and AFM imaging. A drop of $50 \mu \mathrm{l}$ of imaging buffer (20 mM TRIS, $150 \mathrm{mM} \mathrm{KCl,} \mathrm{pH} \mathrm{7.4)} \mathrm{was} \mathrm{injected} \mathrm{into} \mathrm{the} \mathrm{cantilever} \mathrm{holder}$ of the head to create a hanging droplet. The lasers were aligned to the cantilever (BL-AC10DS (Olympus)). Afterwards, the head was placed onto the scanner, directly after cleaving the glued-on $3 \mathrm{~mm}$ Muscovite Mica disk. Due to the heat generated by the readout electronics, the sample temperature was $\sim 6^{\circ} \mathrm{C}$ above ambient. The environment was kept at $6-10^{\circ} \mathrm{C}$ by placing the microscope base, scanner and head into a low-vibration cooler (WL450F-220-FL (Swisscave)) to match the temperature that was used previously to assemble SAS- 6 rings $^{37}$. The head was turned on and left to equilibrate for $15-30 \mathrm{~min}$ to allow the system to stabilize. The surface was approached in static deflection mode using a NanoScope 5 controller. The piezo was then soft retracted and the $z$-piezo control was switched to the home-built controller for PORT imaging or to a digital high-speed lock-in amplifier (UHFLI (Zurich Instruments) for HS-AM-AFM.

The concentrated sample was diluted in imaging buffer to a concentration of $175 \mu \mathrm{g} \mathrm{ml}^{-1}$. This diluted SAS-6 sample was directly injected to reach a final concentration of $3.5 \mu \mathrm{g} \mathrm{ml}^{-1}$. Imaging buffer $(15 \mu \mathrm{l})$ was preloaded into the Hamilton syringe to compensate for the dead volume in the liquid cell.

All the high-speed scanning shown was performed at a $100 \mathrm{~Hz}$ line rate at 512 pixels $\times 256$ lines, which corresponds to 2.56 sframe $^{-1}$. To avoid systematic measurement inaccuracies that might result from spatial or temporal drift of the drive laser, which changes the effective amplitude of the PORT motion, we automatically performed a background correction after each frame.

HS-AM-AFM imaging was done with an amplitude of 1-4 nm. The AM-AFM parameters used to obtain the data in Fig. 1c were $3 \mathrm{~nm}$ of free amplitude at a $500 \mathrm{kHz}$ drive by photothermal excitation, a nominal set point of $2.6 \mathrm{~nm}(86 \%$ of the free amplitude) and $P$ and $I$ gains of the controller adjusted as high as possible such that no oscillation occurred. The cantilever had a $f_{\text {res }}$ of $505 \mathrm{kHz}$ and a spring constant of $k=0.21 \mathrm{~N} \mathrm{~m}^{-1}$. PT-ORT was operated at motion amplitudes of $15-50 \mathrm{~nm}$ with a distance set point of $350-600 \mathrm{pm}$, which corresponds to a force set point of $70-120 \mathrm{pN}$ static force.

Cantilever tips were electron-beam deposited from a carbon precursor onto the existing silicon nitride tips to reduce adhesion of the protein to the lever and to reuse the cantilevers.

Force curve measurement and calculation. Deflection traces for the force calculations in Fig. 3 were acquired on a freshly cleaved mica surface (SPI Supplies) in buffer (imaging buffer, $20 \mathrm{mM}$ TRIS pH 7.4 with $150 \mathrm{mM} \mathrm{KCl}$ ). The use of mica as a substrate for determining the impact force differences between PORT and HS-AM-AFM allows for a consistent and uniform sample. In the case of a softer substrate, such as CrSAS- 6 proteins, we expect the absolute magnitude of the impact forces to decrease, but the relative difference between the two modes to be conserved.

AM-AFM deflection traces were measured and averaged using the Periodic Waveform Analyser function of the high-speed lock-in amplifier (UHFLI (Zurich Instruments)). PORT deflection traces were measured and averaged using the home-built PORT controller. To extract the force curves, the second derivative was calculated and multiplied by the effective mass of the cantilever ${ }^{19}$.

Image processing and data analysis. Images were processed in Gwyddion using an in-house-developed batch-processing script to process large amounts of images automatically. Images were corrected for scanline mismatch (median of differences row alignment) and the background removed (flatten base ${ }^{38}$ ). For better visibility, a 2 pixel conservative denoising filter and a 0.8 pixel Gaussian filter were applied. Afterwards, the pixels were squared and the images exported in a Portable Network Graphics format. Finally, the movies were assembled from individual frames using ffmpeg.

Data availability. The data that support the plots within this paper and other findings of this study are available from the corresponding author upon reasonable request.

\section{References}

36. Nievergelt, A. P., Adams, J. D., Odermatt, P. D. \& Fantner, G. E. Highfrequency multimodal atomic force microscopy. Beilstein J. Nanotechnol. 5, 2459-2467 (2014).

37. Guichard, P. et al. Cell-free reconstitution reveals centriole cartwheel assembly mechanisms. Nat. Commun. 8, 14813 (2017).

38. Erickson, B. W., Coquoz, S., Adams, J. D., Burns, D. J. \& Fantner, G. E. Large-scale analysis of high-speed atomic force microscopy data sets using adaptive image processing. Beilstein J. Nanotechnol. 3, 747-758 (2012). 